\title{
Extension of observed flood series by combining a distributed hydro- meteorological model and a copula-based model
}

\author{
Ana I. Requena Isabel Flores Luis Mediero Luis Garrote
}

\begin{abstract}
Long flood series are required to accurately estimate flood quantiles associated with high return periods, in order to design and assess the risk in hydraulic structures such as dams. However, observed flood series are commonly short. Flood series can be extended through hydro-meteorological modelling, yet the computational effort can be very demanding in case of a distributed model with a short time step is considered to obtain an accurate flood hydrograph characterisation. Statistical models can also be used, where the copula approach is spreading for performing multivariate flood frequency analyses. Nevertheless, the selection of the copula to characterise the dependence structure of short data series involves a large uncertainty. In the present study, a methodology to extend flood series by combining both approaches is introduced. First, the minimum number of flood hydrographs required to be simulated by a spatially distributed hydro-meteorological model is identified in terms of the uncertainty of quantile estimates obtained by both copula and marginal distributions. Second, a large synthetic sample is generated by a bivariate copula-based model, reducing the computation time required by the hydro-meteorological model.
\end{abstract}

The hydro-meteorological modelling chain consists of the RainSim stochastic rainfall generator and the Real-time Interactive Basin Simulator (RIBS) rainfall-runoff model. The proposed procedure is applied to a case study in Spain. As a result, a large synthetic sample of peak-volume pairs is stochastically generated, keeping the statistical properties of the simulated series generated by the hydro-meteorological model. This method reduces the computation time consumed. The extended sample, consisting of the joint simulated and synthetic sample, can be used for improving flood risk assessment studies.

Keywords Flood stochastic generation - Copulas . Rainfall-runoff modelling $\cdot$ Short data series $\cdot$ Flood frequency analysis

\section{Introduction}

Estimates of flood quantiles for high return periods are essential for designing and assessing flood risk in hydraulic structures such as dams. Such quantiles are usually estimated by flood frequency analyses. There are many studies throughout the literature that involve univariate flood frequency analyses, usually focused on the study of the peak flow (e.g., Cunnane 1989; GREHYS 1996). Nevertheless, the multivariate nature of floods requires a multivariate flood frequency analysis (Chebana and Ouarda 2011) for certain applications. Lately, bivariate approaches studying the peak flow and hydrograph volume jointly have become widespread (e.g., Goel et al. 1998; Yue et al. 1999; Favre et al. 2004; Shiau et al. 2006). The more complex trivariate approach is considered in some studies by including the duration of the hydrograph (e.g., Serinaldi and Grimaldi 2007). 
Long flood series are required to obtain accurate estimates of quantiles associated with high return periods (Saad et al. 2015), which in the case of a multivariate flood frequency analysis is even more important because of a higher uncertainty derived from a larger number of parameters involved in the study. Nevertheless, the available flood data series are short in practice, commonly between 30 and 80 years (e.g., Zhang and Singh 2007; Klein et al. 2010; Requena et al. 2015b). The need for extending observed data series to perform a proper flood frequency analysis can be addressed by either: (i) simulation via hydro-meteorological modelling, reproducing the catchment response by using long (observed or synthetic) rainfall series; or (ii) stochastic generation via a statistical analysis, such as by a multivariate model that represents the joint distribution of the studied variables.

Regarding hydro-meteorological simulation, Beven (1987) first proposed the idea of coupling a stochastic rainfall generator and a rainfall-runoff model to reproduce the flood frequency curve in a Welsh catchment, following the theoretical work presented by Eagleson (1972). Later, Cameron et al. (1999) elaborated on the idea of calibrating the predicted flood frequency curve by a model through the observed flood series for small return periods and using it to extrapolate flood magnitudes for larger return periods. Blazkova and Beven $(1997,2004)$ applied the procedure to several Czech catchments for dam safety evaluation. Calver and Lamb (1995) evaluated the proposed approach in ten catchments in the UK. Similar methodologies have been applied in Australia, (Boughton et al. 2002), US (England et al. 2007), France (Paquet et al. 2013), Norway (Lawrence et al. 2014), Russia (Kuchment et al. 2003), South Africa (Chetty and Smithers 2005) and other countries (Boughton and Droop 2003). These approaches are based on combining a stochastic rainfall generator and a hydrological model that reproduces the rainfall-runoff response in the catchment (Vrugt et al. 2002; Engeland et al. 2005). Such hydrological models can be classified into distributed or lumped, depending on whether the parameter values are spatially distributed or averaged in the catchment; and continuous or event-based, depending on whether a long time series, usually with a daily time step, or independent flood events, usually with around an hourly time step, are simulated. The underlying assumption is that a hydrological model calibrated with the observed data is able to simulate a set of feasible flood hydrographs that can be generated in a catchment, using synthetic rainfall events and the catchment characteristics as input. The main advantage of this approach is to provide not only the statistical characterisation of extreme values for the relevant variable, but also an ensemble of hydrographs that can force the structure under design, thus allowing for a better performance characterisation. A distributed event-based model with a high temporal and spatial resolution is required to represent correctly the variability of flood generation processes in the catchment. However, the higher is the model resolution, the longer is the computation time. Therefore, the required computation time can prevent the generation of arbitrarily long series with a good characterisation of the catchment response.

A multivariate distribution can be used for extending the available flood series, stochastically generating a larger series that keeps the statistical properties of the original sample and allows obtaining quantiles for high return periods. The shortcomings of the traditional multivariate distributions, such as the need for using the same marginal distribution for characterising all variables involved in the analysis, and the assumption of a linear relation between them, are overcome by using copulas (e.g., Salvadori et al. 2007). The use of copulas in hydrology and specially in multivariate flood frequency analysis is increasingly spreading (e.g., De Michele et al. 2005; Zhang and Singh 2006; Song and Singh 2010; Requena et al. 2013; Zhang et al. 2013). The multivariate distribution of several random variables can be obtained via the marginal distribution of each variable and a copula function, which is a multivariate distribution with uniform margins that characterises the dependence structure between them. The main advantages of the stochastic generation of flood data by a multivariate distribution based on copulas are twofold: (i) they only need a flood series as input; and (ii) the computation time required once the multivariate distribution is fitted is negligible. The drawback resides in the difficulty of properly selecting and fitting the multivariate distribution when the available data length is short. In this case, several copula families usually pass the goodness-of-fit test and a larger uncertainty is involved in fitting the parameters, which leads to larger uncertainties in estimates of the right tail of both copula and marginal distributions.

Some studies dealt with the idea of considering both approaches. Candela et al. (2014) applied a bivariate Archimedean copula-based distribution for characterising rainfall duration and intensity, in order to generate single synthetic rainfall events to be used as input in a conceptual fully distributed rainfall-runoff model based on the curve number method. The copula approach was then applied to the peak flow and hydrograph volume series of 5000 events synthetically generated from such a procedure, to obtain the flood design hydrograph related to a given joint return period. Klein et al. (2010) used 10,000 flood hydrographs generated from a distributed hydro-meteorological model as initial data for developing a copula-based flood frequency analysis in which dam safety was assessed. Dam safety was also evaluated by Giustarini et al. (2010), analysing the water level reached at a given dam by three sets of synthetic flood hydrographs. The first and second sets 
were obtained by generating peak-volume pairs from an Archimedean copula-based distribution fitted to observed data, and to several 1000-length synthetic data generated from a continuous hydro-meteorological model, respectively. The third set consisted of flood hydrographs generated directly from the continuous model. Damovertopping results were of the same order of magnitude for the three sets, although more dangerous events were obtained by the second set. On the basis of the drawbacks and advantages regarding the generation of each set, the notion of combining approaches was highlighted.

A long sample length was arbitrarily generated via hydro-meteorological modelling in the three aforementioned studies. The main aim of the present study is to determine the minimum number of flood hydrographs needed to be simulated by a hydro-meteorological model, in order to be used as input for a copula-based distribution. This is motivated by the need of obtaining a large synthetic series in short time, as the hydro-meteorological model is computationally very demanding because of entailing a high spatial and temporal resolution. The longer simulated sample improves the fitting of the distribution, as observed series are usually short and the hydro-meteorological model simulates the variability in the catchment response. Then, the flood series can be extended again by stochastically generating an (arbitrarily) long sample by the fitted copula-based distribution. The proposed mixed approach addresses the need for extending short observed peakvolume series, combining the ability to simulate the feasible catchment responses by a distributed rainfall-runoff model and the computational efficiency offered by statistical models. The hydro-meteorological modelling chain used in the present study consists of the RainSim stochastic rainfall generator, and the Real-time Interactive Basin Simulator (RIBS) hydrological rainfall-runoff model. The RainSim model is a spatial-temporal stochastic rainfall generator (Burton et al. 2008), while the RIBS model is an event-based distributed rainfall-runoff simulator of the catchment response under rainfall events that are spatially distributed (Garrote and Bras 1995a, b). The structure of the present paper is divided into the following sections: Methodology is presented in Sect. 2, Application consisting of the case study and results is shown in Sect. 3 and Conclusions are summarised in Sect. 4.

\section{Methodology}

The present study focuses on a bivariate analysis of floods by using the maximum peak flow $(Q)$ and its associated hydrograph volume $(V)$. The methodology consists of the following steps (see Fig. 1 for an overview): (i) simulation of a set of flood hydrographs by a hydro-meteorological model calibrated with observed flood series, using synthetic rainfall series from a stochastic rainfall generator; (ii) sensitivity analysis to identify the minimum data length needed for keeping the statistical properties of the whole simulated data series when the marginal distribution and copula candidates are fitted; (iii) identification of the bivariate model based on copulas consisted of the marginal distribution that best fits each univariate variable and the copula that best represents the dependence structure between them, as well as the corresponding minimum data length to be fitted; and (iv) validation of the methodology by comparing the flood frequency curve (of each marginal distribution) and the copula level curves of a large sample simulated by the hydro-meteorological model, with those of a set of synthetic samples generated with the same size by the proposed mixed-approach. That is, synthetic samples generated via the bivariate distribution fitted to samples from the hydro-meteorological model with the data length identified in step (iii). Moreover, as an illustration of the results obtained by the application of the procedure, joint return period curves estimated by using the large simulated sample are compared with those obtained by a given synthetic sample. The proposed methodology allows reducing the computation time, while maintaining the statistical properties of the flood series simulated by the hydro-meteorological model. The methodology is applied to the Santillana reservoir catchment in the Manzanares River located in Spain.

\subsection{Simulation of flood hydrographs by a hydro- meteorological model}

A set of flood hydrographs is generated by the hydro-meteorological modelling chain consisting of the RainSim rainfall simulator and the RIBS rainfall-runoff model. The RainSim V3 model is a stochastic rainfall generator based on the spatial-temporal Neyman-Scott rectangular pulses (NSRP) model (Cowpertwait 1994, 1995). This model allows the simulation of continuous series of rainfall of a number of years for a set of rain gauges in the catchment and with an arbitrary time step. The model details are described in Burton et al. (2008). The RIBS model simulates the catchment response to spatially distributed rainfall events and results in flood events at the catchment discharge point (Garrote and Bras 1995a, b). The RIBS model consists of two modules. The first is a runoff-generation module and the second simulates the runoff propagation. The runoff generation depends on the calibration parameter $f\left(\mathrm{~mm}^{-1}\right)$ that controls the decrease of saturated hydraulic conductivity with depth and the soil properties that have to be defined for each soil class. These properties are the saturated hydraulic surface conductivities in directions normal and parallel to the surface, the residual soil 


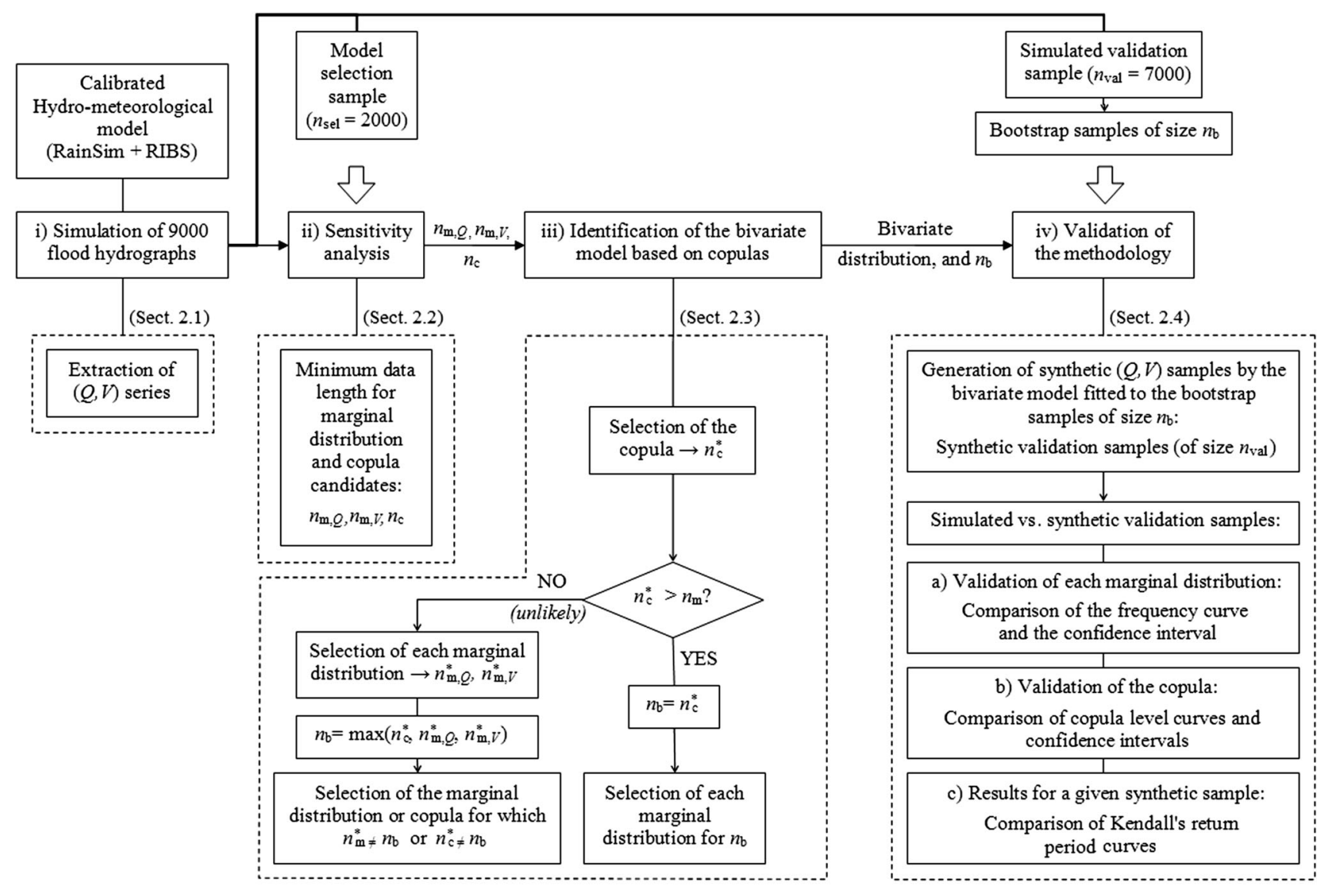

$n_{\text {sel }}$ : sample length of the model selection sample $n_{\mathrm{val}}$ : sample length of the simulated validation sample $n_{\mathrm{b}}$ : minimum sample length for the bivariate distribution

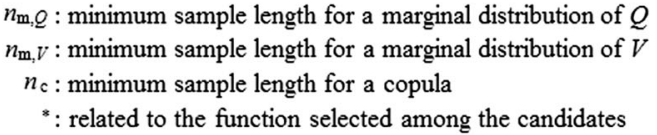

$n_{\mathrm{m}, Q}:$ minimum sample length for a marginal distribution of $Q$ $n_{\mathbf{m}, V}:$ minimum sample length for a marginal distribution of $V$ $n_{\mathrm{c}}$ : minimum sample length for a copula

${ }^{*}$ : related to the function selected among the candidates

Fig. 1 Diagram of the steps forming the proposed methodology

moisture content, the saturated moisture content and the index of soil porosity (Cabral et al. 1992). The runoff propagation depends on the hill-slope and the riverbed velocities. The latter is proportional to the coefficient $C_{v}$ $\left(\mathrm{m} \mathrm{s}^{-1}\right)$ that characterises the relation between riverbed velocity and discharge at the catchment outlet. Both velocities are considered uniform throughout the catchment at any time, and defined by their relationship to the dimensionless parameter $K_{v}$. Event-based models need an estimate of the initial moisture content in the catchment at the beginning of the flood event. In the case of the RIBS, it corresponds to the water table depth that is in long term equilibrium with a constant recharge rate.

Once a large set of flood hydrographs is simulated, the associated $Q-V$ series is extracted by identifying the maximum peak flow and the hydrograph volume (see Sect. 3.2.1). Such simulated $Q-V$ series is divided into two samples: the model selection sample with a sample length $n_{\text {sel }}$, for performing steps (ii) and (iii); and the simulated validation sample with a sample length $n_{\mathrm{val}}$, for carrying out step (iv). At this point it is important to verify if, as expected, the studied variables $Q$ and $V$ are dependent, in which case the joint analysis (by the marginal distributions and copula) is needed. This is done by the rank-based nonparametric Kendall's tau $(\tau)$ measure, through which the independence between variables is rejected if the associated $p$-value is less than 0.05 (Genest and Favre 2007).

\subsection{Sensitivity analysis: minimum data length needed}

A prior step to the selection of the bivariate distribution of the $Q-V$ series is the identification of the minimum data length $(n)$ necessary for both marginal distribution and copula fits to be robust enough in terms of uncertainty of estimates. When marginal distributions are considered, the 
variable chosen for performing the sensitivity analysis is the univariate quantile $\left(q_{T}\right)$ for a given return period value (T). Note that $T$ is the inverse of $(1-p)$, where $p$ is the non-exceedance probability of $q_{T}$.

In the case of using copulas, the bivariate quantile is a curve in the $Q-V$ space instead of a single value like in the univariate case. However, a single-value variable is needed for conducting the sensitivity analysis. The Kendall's return period (Salvadori and De Michele 2004; Salvadori et al. 2011) could be a suitable variable to be used as a surrogate of the bivariate quantile, as each bivariate quantile curve is associated with a given Kendall's return period value that depends on the copula. Moreover, the Kendall's return period is the joint return period that provides an analogous definition of quantile to that considered in the univariate approach (Salvadori and De Michele 2010). Nevertheless, a long computation time is needed for performing the sensitivity analysis based directly on this variable. Consequently, as the aim of the proposed method is to reduce the computation time, the copula parameter $(\theta)$ is chosen to conduct the sensitivity analysis on the bivariate series. It should be noted that $\theta$ is needed for estimating the Kendall's return period. In summary, the minimum required $n$ is obtained by analysing the univariate quantile associated with a given $T, q_{T}$, for marginal distributions, and the copula parameter, $\theta$, for bivariate copulas.

The proposed procedure is the following: (i) 1000 bootstrap samples of varying length $n=25,50, \ldots, 1000$ are obtained from the model selection sample of length $n_{\text {sel }}$, without replacement; (ii) both $q_{T}$ and $\theta$ are estimated for the 1000 bootstrap samples associated with each $n$, considering the set of candidate marginal distributions and copulas; (iii) the sample distribution of either $q_{T}$ or $\theta$ for each $n$ is displayed in a box plot where the 25th and 75th percentiles are shown as the borders of the box, hereafter such a statistical interval is named as confidence interval; (iv) the minimum length required for each either univariate distribution function (named as $n_{\mathrm{m}}$ ) or copula (named as $n_{\mathrm{c}}$ ) is determined as the smallest $n$ for which the confidence interval of the bootstrap samples lies within boundaries related to the model selection sample. These boundaries are the sampling confidence interval of the model selection sample increased by $5 \%$, assuming that an increase of $5 \%$ in the uncertainty of estimates at the expense of reducing the record length is acceptable. In the case of the marginal distributions, the sampling confidence interval is obtained by generating 10,000 samples by the distribution function fitted to the model selection sample, calculating the value of $q_{T}$ for each sample and obtaining the 25th and 75th percentiles. Note that in the case of copulas, only 1000 bootstrap samples are generated to avoid a long computation time for generating such a confidence interval in terms of the copula parameter $\theta$.
As a result, the value of $n_{\mathrm{m}}$ for each marginal distribution (named as $n_{\mathrm{m}, Q}$ for $Q$ and $n_{\mathrm{m}, V}$ for $V$ ) as well as the value of $n_{\mathrm{c}}$ for each copula is obtained. Note that in the case of two-parameter copulas, $n_{\mathrm{c}}$ is identified as the maximum of the two values obtained by applying the procedure to each parameter. Also note that $n_{\mathrm{m}}$ can be slightly different for a same distribution function for each variable, as the sampling uncertainty depends on its distribution parameters.

\subsection{Identification of the bivariate model based on copulas}

The bivariate model for accomplishing the stochastic generation of large $Q-V$ samples is based on the Sklar's Theorem (Sklar 1959), through which the bivariate joint cumulative distribution of the variables $Q$ and $V, H(q, v)$, is obtained as:

$H(q, v)=C\left(F_{Q}(q), F_{V}(v)\right), \quad q, v \in \Re$,

where $F_{Q}($.$) is the cumulative marginal distribution of Q, q$ is a given value of such a variable (the same holds for $V$ ), and the copula function $C\left(u_{1}, u_{2}\right):[0,1]^{2} \rightarrow[0,1]$ is a bivariate joint cumulative distribution with uniform marginal distributions that can be expressed by using $u_{1}$ $=F_{Q}(q)$ and $u_{2}=F_{V}(v)$. Thus, the estimate of $H(q, v)$ requires the identification of the marginal distributions that best represent the univariate $Q$ and $V$ variables, as well as the copula that best characterises the dependence structure between them. The minimum sample length for fitting such a bivariate distribution, $n_{\mathrm{b}}$, is determined as the maximum of the minimum required lengths for fitting the marginal distributions, $n_{\mathrm{m}, Q}$ and $n_{\mathrm{m}, V}$, and the copula, $n_{\mathrm{c}}$, i.e., $n_{\mathrm{b}}=\max \left(n_{\mathrm{m}, Q}, n_{\mathrm{m}, V}, n_{\mathrm{c}}\right)$. The procedure for identifying the bivariate distribution begins with the selection of the copula, since the copula is expected to require a larger data length than the marginal distributions because of its bivariate nature. As a result, the marginal distributions and copula to use for obtaining the bivariate distribution of $Q$ and $V$ are selected, and $n_{\mathrm{b}}$ is identified.

\subsubsection{Selection of the copula}

The best copula is selected by considering 1000 bootstrap samples of the corresponding length $n_{\mathrm{c}}$ obtained in Sect. 2.2, for each copula. One-parameter copulas, such as the Clayton, Frank, Gumbel and Plackett copula, and twoparameter copulas, such as the BB1 copula (Joe 1997), are considered as copula candidates in the present study.

The selection of the best copula among the candidates is not straightforward and different criteria should be considered (Chowdhary et al. 2011; Requena et al. 2013). Because of the nature of the present study, the procedure 
for selecting the best copula is based on three criteria, for which results are drawn in a box plot for each copula: the fit of the copula to the data, the adequacy of the estimate of a high Kendall's return period value that is directly related to the bivariate quantile estimate, and the results of a model selection criterion that allows ranking the copulas.

The first criterion, related to the ability of the copula to characterise the data, is performed under the goodness-offit test based on the Cramér-von Mises statistic (Genest et al. 2009), $S_{n}$ :

$S_{n}=\sum_{i=1}^{n_{\mathrm{c}}}\left\{C_{n}\left(\frac{R_{i}}{n_{\mathrm{c}}+1}, \frac{S_{i}}{n_{\mathrm{c}}+1}\right)-C_{\theta}\left(\frac{R_{i}}{n_{\mathrm{c}}+1}, \frac{S_{i}}{n_{\mathrm{c}}+1}\right)\right\}^{2}$,

where $\left(R_{i}, S_{i}\right)$ are the ranks of the $\left(Q_{i}, V_{i}\right)$ pairs of each bootstrap sample, $C_{\theta}$ is the copula fitted to such data estimating the parameter(s) by the maximum pseudo-likelihood method (Genest et al. 1995) and $C_{n}$ is the empirical copula:

$$
\begin{aligned}
C_{n}\left(u_{1}, u_{2}\right) & =\frac{1}{n_{\mathrm{c}}} \sum_{i=1}^{n_{\mathrm{c}}} 1\left(\frac{R_{i}}{n_{\mathrm{c}}+1} \leq u_{1}, \frac{S_{i}}{n_{\mathrm{c}}+1} \leq u_{2}\right), \quad u_{1}, u_{2} \\
& \in[0,1],
\end{aligned}
$$

where $1(\mathrm{~A})$ is the indicator function of the set $\mathrm{A}$ (that equals 1 inside $\mathrm{A}$ and 0 otherwise). $S_{n}$ is indeed an error measure between the values of the empirical copula and those obtained by the fitted copula. Thus, the smaller is the (median) $S_{n}$ value, the better is the copula. The $p$-value associated with $S_{n}$, estimated by a parametric bootstrap procedure (Genest and Remillard 2008) where 1000 simulations are employed in the present study because of computation time limitations, is also obtained to carry out the goodness-of-fit test itself. A copula is formally accepted when its (median) $p$-value is greater than 0.05 . However, as it happens with other statistical tools, it is expected not to obtain suitable $p$-values in case of a large sample size is considered (see Vandenberghe et al. 2010; Requena et al. 2015a). In addition, a formal goodness-of-fit test is not performed in the copula selection process in some studies (e.g., Klein et al. 2010). In fact, the $p$-value is very useful to test if a sample comes from a given copula. However, in practice, observed flood series could not follow any of the existing copulas, due to the natural variability of floods. Consequently, the copula that best characterises the observed sample should be found, instead of the true copula that could represent the observed sample perfectly, as in some cases it does not exist.

The second criterion is related to the adequacy of the Kendall's return period estimated by the copula, for a large copula value $t \in[0,1]$, which is based on the Kendall's function $K_{C}(t)=P\left[C_{\theta}\left(u_{1}, u_{2}\right) \leq t\right]$ (Genest and Rivest 1993). Its theoretical estimate $\left(T_{K}\right)$ is expressed as:

$T_{K}=\frac{1}{1-K_{\mathrm{C}}(t)}$

The Kendall's function has an analytical expression for Archimedean copulas (e.g., the Clayton, Frank and Gumbel copula), but simulation is required for the rest of copula families (Salvadori et al. 2011). The issue is that extreme value copulas (e.g., the Gumbel and Galambos copula) have associated the same Kendall's function (Genest et al. 2006) and therefore the criteria based on the Kendall's function is not able to distinguish among them. The value of the Kendall's return period is also estimated and the results of each copula are plotted. In this case, the best copula is that with the closest (median) theoretical Kendall's return period to the empirical Kendall's return period $\left(\dot{T}_{K}\right)$, estimated by the Kendall's function associated with the empirical copula of the whole model selection sample.

The third criterion is the Akaike Information Criterion (AIC) (e.g., Zhang and Singh 2006).

$\mathrm{AIC}=-2 \sum_{i=1}^{n_{\mathrm{c}}} \ln \left[c_{\theta}\left(\frac{R_{i}}{n_{\mathrm{c}}+1}, \frac{S_{i}}{n_{\mathrm{c}}+1}\right)\right]+2 k$,

where $c_{\theta}($.$) is the density function of the fitted copula, and$ $k$ is the number of copula parameters. The best copula for this criterion is that with the smallest (median) AIC value. The AIC penalises the copulas with more parameters, as it can be seen through the second term of its formula.

Note that although the median is the value considered to assess the performance of each criterion, the variability in the results (i.e., the height of the boxes) should also be considered in the decision process, as it makes reference to the uncertainty in the results given by the copula. As a result of taking into account all the information provided by these criteria, the best copula is selected and its minimum required sample length, $n_{\mathrm{c}}^{*}$, is identified. As illustration and in order to provide a visual support of the behaviour of the copulas, the empirical (i.e., based on $C_{n}$ ) and theoretical estimate of the Kendall's function for the model selection sample is also provided.

\subsubsection{Selection of the marginal distributions}

If the minimum sample length required for the selected copula $n_{\mathrm{c}}^{*}$ is greater than any of the $n_{\mathrm{m}}$ values obtained in Sect. 2.2, the minimum sample length required by the bivariate distribution is given by that of the copula, i.e., $n_{\mathrm{b}}=n_{\mathrm{c}}^{*}$ and hence, the selection of the marginal distributions is done under 1000 bootstrap samples of size $n_{\mathrm{b}}$. Distributions usually used in hydrology, such as the 
Gumbel $(\mathrm{G})$, generalised extreme value (GEV), generalised logistic (GLO) and log-normal (LNO) distribution, are the marginal distributions selected as candidates. Because of the nature of this study, the best marginal distribution is identified as that with the smallest distance between the median quantile estimate obtained by a given marginal distribution, $\hat{q}_{T}$ (named as $\hat{q}_{T}^{Q}$ for $Q$ and $\hat{q}_{T}^{V}$ for $V$ ), and the median quantile estimate obtained by the empirical distribution, $\dot{q}_{T}$, both assessed by using 1000 bootstrap samples of size $n_{\mathrm{b}}$. Such a distance is expressed as the relative error $(R E)$ in percentage:

$R E=100 \frac{\operatorname{median}\left(\hat{q}_{T}\right)-\operatorname{median}\left(\dot{q}_{T}\right)}{\operatorname{median}\left(\dot{q}_{T}\right)}$

As a result, the marginal distribution with the smallest absolute value of $R E$ is selected for each variable ( $Q$ and V).

Note that in the (more unlikely) case that $n_{\mathrm{c}}^{*}$ is less than some $n_{\mathrm{m}}$, the selection of the marginal distributions is conducted by using 1000 bootstrap samples of the corresponding size $n_{\mathrm{m}}$. Then, as $n_{\mathrm{b}}=\max \left(n_{\mathrm{m}, Q}^{*}, n_{\mathrm{m}, V}^{*}, n_{\mathrm{c}}^{*}\right)$, the selection process should be repeated for the marginal distribution or copula for which $n_{\mathrm{m}, Q}^{*}, n_{\mathrm{m}, Q}^{*}$ or $n_{\mathrm{c}}^{*}$ is different from $n_{\mathrm{b}}$ (see Fig. 1 for a diagram of the process). As illustration, the fit of the marginal distributions to the model selection sample is also provided to visually check how the marginal distributions behave regarding the whole sample.

\subsection{Validation of the methodology}

The aim of this section is to check the adequacy of the proposed methodology by comparing the behaviour of a large sample obtained directly through the hydro-meteorological model (i.e., the simulated validation sample introduced in Sect. 2.1), with samples of the same length (called synthetic validation samples) stochastically generated by fitting the selected bivariate distribution to smaller samples of size $n_{\mathrm{b}}$ belonging to such a simulated validation sample. The present section consists of the procedure needed to generate synthetic samples by the bivariate copula-based distribution, followed by the validation of the marginal distributions, the validation of the copula, and an example of the results provided by the application of the methodology in comparison to those obtained by only using the hydro-meteorological model.

The generation of synthetic validation samples is conducted by using the bivariate distribution identified in Sect. 2.3 based on the information provided by the model selection sample, and small bootstrap samples obtained from the simulated validation sample. The procedure is described below: (i) a bootstrap $Q-V$ sample of size $n_{\mathrm{b}}$ is obtained from the simulated validation sample without replacement; (ii) the selected copula is fitted to the bootstrap sample, generating a synthetic sample of size $n_{\text {val }}$ consisted of $\left(u_{1}, u_{2}\right)$ pairs; (iii) the selected marginal distribution of $Q$ and $V$ are used for transforming the $\left(u_{1}, u_{2}\right)$ pairs into $(Q, V)$ pairs formed by the components $q=F_{Q}^{-1}\left(u_{1}\right)$ and $v=F_{V}^{-1}\left(u_{2}\right)$, where $F_{Q}^{-1}\left(\right.$.) and $F_{V}^{-1}($.) are the inverses of the marginal distributions of $Q$ and $V$, respectively. This synthetic $Q-V$ sample of size $n_{\text {val }}$ is called synthetic validation sample. A new synthetic validation sample is generated each time the process is performed.

The validation of the marginal distributions is performed based on the following procedure: (i) the selected marginal distributions for $Q$ and $V$ are fitted to the whole simulated validation sample, obtaining their flood frequency curve; (ii) the selected marginal distributions are also fitted to each of the 10,000 synthetic validation samples generated by the procedure described above, obtaining their associated flood frequency curves; (iii) the confidence interval (i.e., the statistical interval consisted of the 25th and 75th percentiles) from the 10,000 synthetic flood frequency curves is obtained for given $T$ values; and (iv) such a confidence interval is compared with the flood frequency curve obtained by the simulated validation sample in the first step of the present procedure.

An analogous process is carried out for the validation of the copula: (i) the copula selected is fitted to the whole simulated validation sample, obtaining the copula probability level curve for given $p$-values; (ii) the selected copula is also fitted to each of the 1000 synthetic validation samples generated by the procedure described at the beginning of the present section, obtaining their associated copula probability level curves formed by the $\left(u_{1}, u_{2}\right)$ points that fulfil $C\left(u_{1}, u_{2}\right)=p$, for given probability values $p$. Only 1000 samples instead of 10,000 are used for avoiding a long computation time when the confidence intervals are estimated; (iii) For each $u_{1}$ value, the confidence interval of the $u_{2}$ values for the 1000 synthetic probability curves is obtained for each $p$; and (iv) the confidence interval associated with each probability value $p$ is compared with the copula probability level curve obtained when fitted to the simulated validation sample.

As illustration of the results obtained by applying the proposed methodology, a given synthetic validation sample is plotted together with the simulated validation sample, and the Kendall's return period curves for both samples are also estimated and drawn.

\section{Application}

The case study and the results obtained by the application of the proposed methodology are shown in the present section. 


\subsection{Case study}

The application of the methodology is carried out on the gauging station of the Santillana reservoir in the Manzanares River, which belongs to the Tagus River catchment and is located in the centre of Spain (Fig. 2). The catchment drainage area of the Santillana reservoir gauging station is $325.6 \mathrm{~km}^{2}$. Mean daily outflow discharge and reservoir volume series are available at this gauging station for the period 1958-2002, from which the series of mean daily inflow discharges was extracted. This case study is chosen due to the previously available calibration of the hydro-meteorological model RainSim-RIBS. The rainfall simulator was calibrated

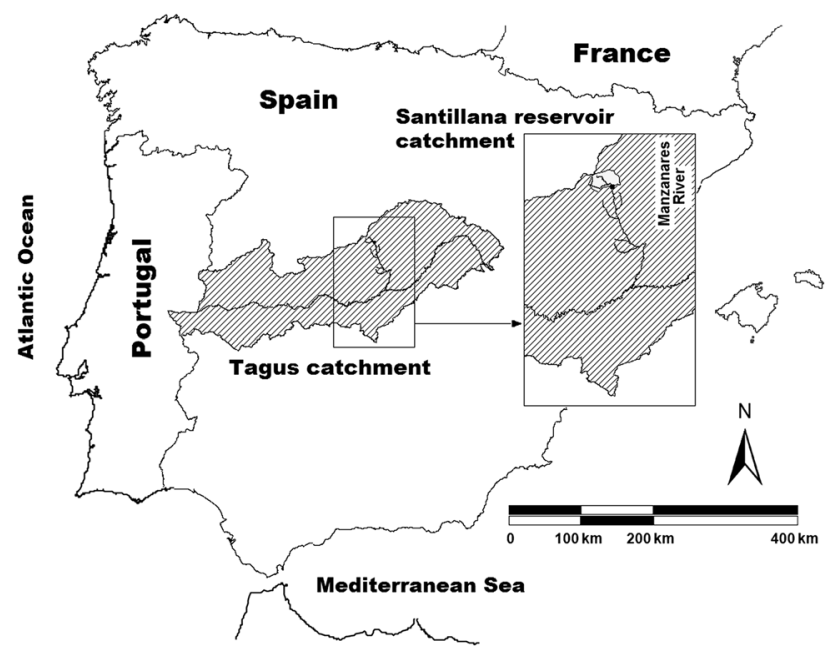

Fig. 2 Location of the case study: catchment of the Santillana reservoir gauging station by Flores et al. (2013) and the rainfall-runoff model by Mediero et al. (2011). The RainSim V3 model was calibrated through a set of observed daily series recorded at 15 raingauges with varying length between 11 and 118 years, where the largest series entails the period 1893-2011. As a result, a 9000-year length hourly rainfall series was generated by the calibrated model at each rain-gauge. The RIBS model was calibrated in the entire Manzanares River catchment with a drainage area of $1248 \mathrm{~km}^{2}$, where the Santillana reservoir catchment is its headwaters. The calibration process is based on a simultaneous minimisation of four objective functions (root mean square error, mean absolute error, coefficient of Nash-Sutcliffe efficiency and time to peak) that account for different hydrograph characteristics, resulting in a probability density function for characterising each of the model parameters subject to calibration. Validation of the probabilistic model was performed via simulations of the calibrated model for each validation event; assessing the bias of the results through a variation of the Nash-Sutcliffe efficiency coefficient, and the accuracy of the results by the inclusion coefficient.

\subsection{Results}

\subsubsection{Simulation of flood hydrographs by a hydro- meteorological model}

Storm events were then simulated individually from the 9000-year length hourly rainfall series generated in the 15 rain-gauges, as RIBS is an event-based model. Independent storm events were identified via the exponential method
Fig. 3 Validation of the hydrometeorological model. Observed data and empirical frequency curves of inflow volume for a one; $\mathbf{b}$ two; c three; and $\mathbf{d}$ four consecutive days
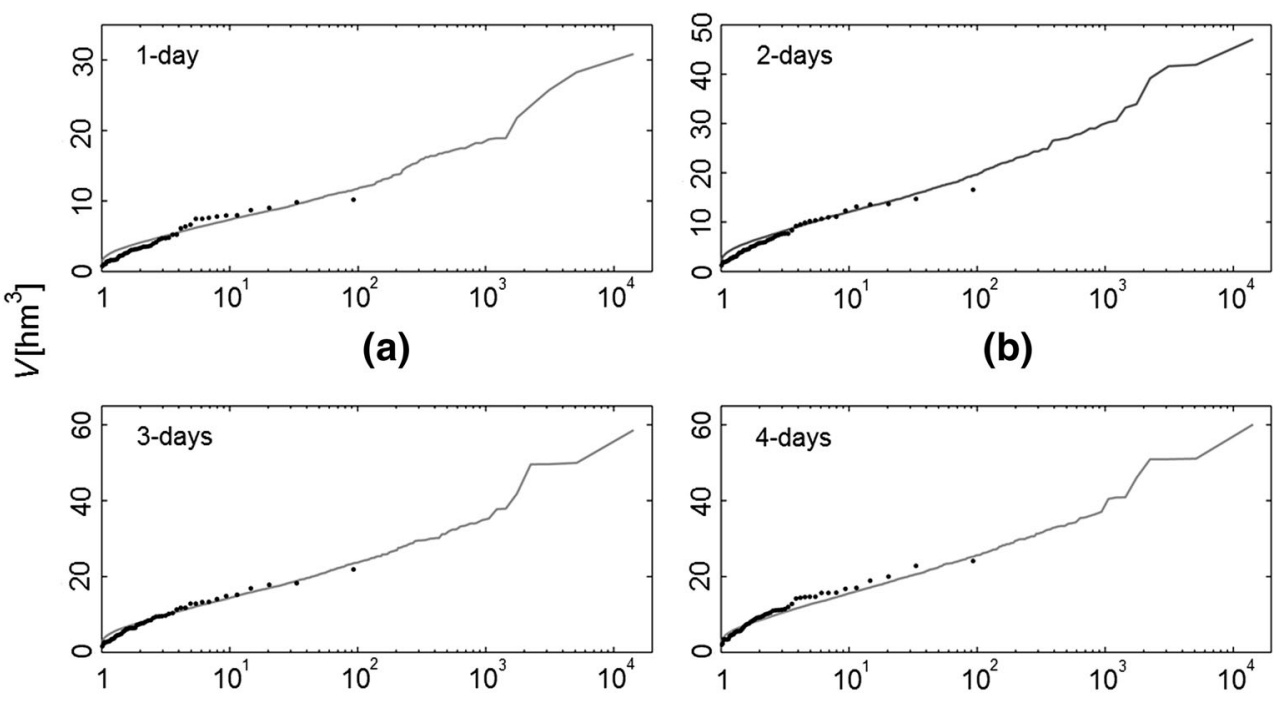

(c)

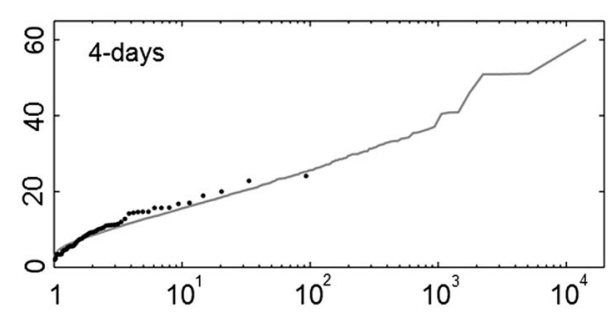

(d)

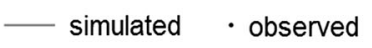




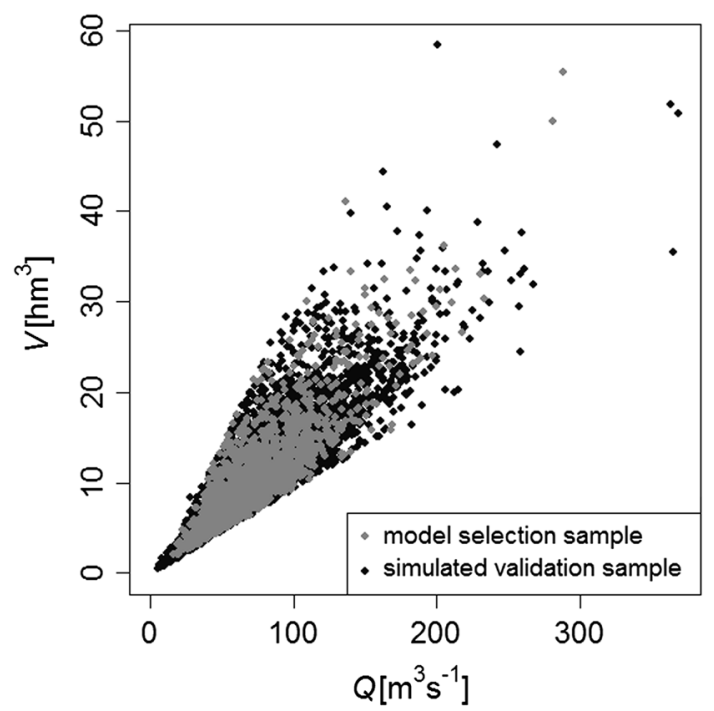

Fig. 4 Scatter plot of the $(Q, V)$ pairs of the samples generated through the hydro-meteorological model divided into the model selection sample and the simulated validation sample

(Restrepo-Posada and Eagleson 1982), fixing a minimum dry period between events, in which rainfall is less than a given threshold (Bonta and Rao 1988). In order to reduce the number of simulations, a specific subset of five events was selected from each year, assuming that the event generating the maximum volume or peak flow hydrograph in a year is included among them.

Consequently, 45,000 synthetic flood events were generated through the calibrated RIBS rainfall-runoff model, accounting for random initial moisture content states among a representative set of 13 initial states in the catchment, in order to generate an ensemble of hydrographs that covers the range of totally dry to completely saturated soils. Each year, the hydrograph with the maximum peak flow was selected and its volume calculated, obtaining 9000 years of synthetic $Q-V$ series. In order to validate the model in terms of the flood frequency curve, the observed and simulated frequency curves for 1-, 2-, 3- and 4-days accumulated inflow volumes were calculated and compared (Fig. 3). Validation was conducted by using inflow volumes for different durations, as information about the instantaneous peak flow of observed inflow hydrographs is not available at this site. It should be noted that the 1-day inflow volume series is used to validate the peak flow of observed inflow hydrographs, as it is characterised by the mean daily discharge. Results show that the model represents suitably the flood frequency curves. Therefore, the hydro-meteorological model can be used for extending the observed series to enable an accurate flood frequency analysis by selecting and fitting the bivariate
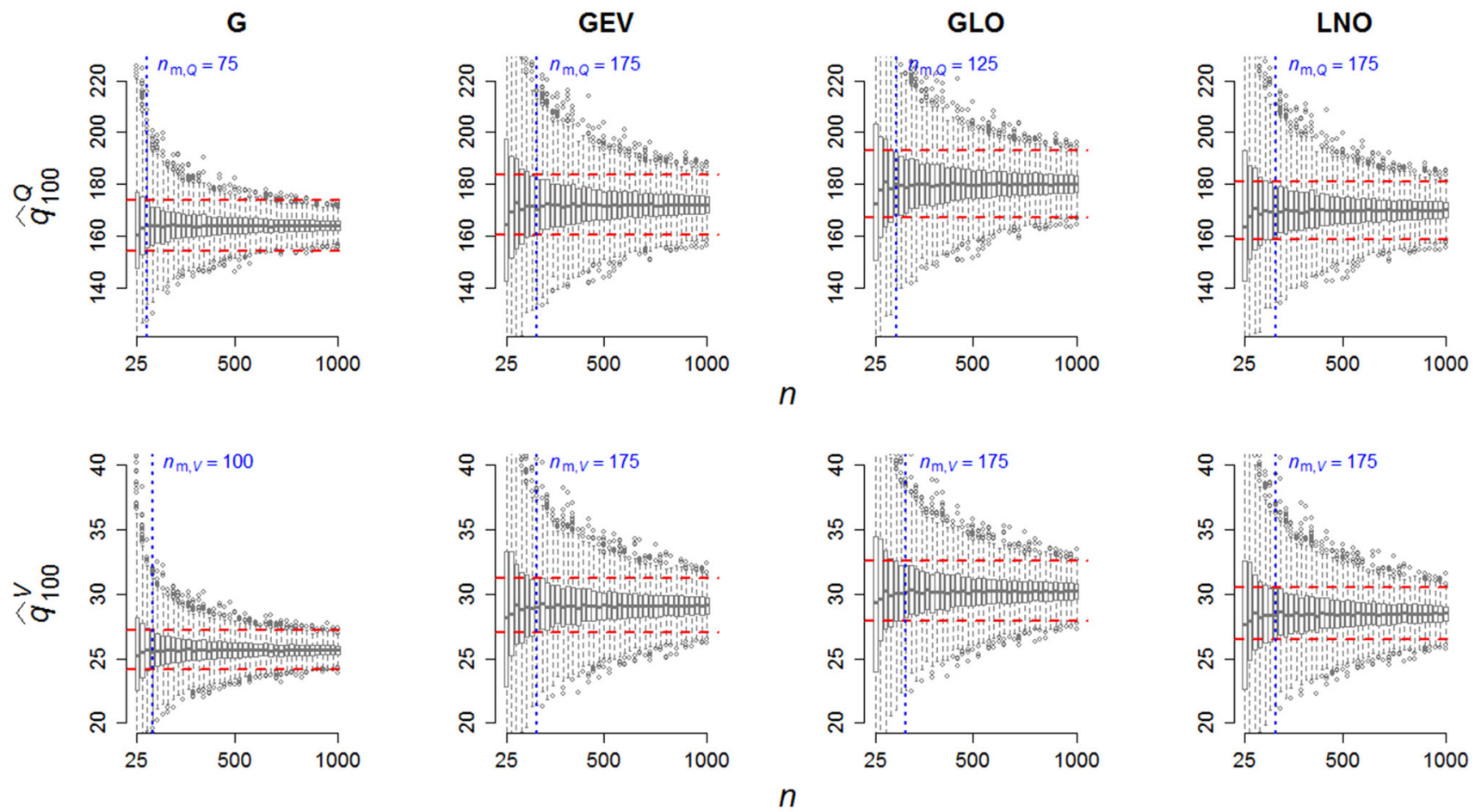

$\overline{-z}$ boundaries related to model selection sample

Fig. 5 Sensitivity analysis for identifying the minimum sample length required for the marginal distributions. The box plot of the quantiles estimated from the bootstrap samples of length $n$ is plotted along the $x$-axis, where the points represent the outliers 
copula-based distribution via the $Q-V$ series extracted from the flood hydrographs simulated by the calibrated hydrometeorological model, instead of via the short-length observed flood data.

As a result, the 9000 synthetic $Q-V$ pairs obtained through the hydro-meteorological model, entailing a 1-h temporal and 100-m spatial resolution, were used. The computation time needed for its generation was approximately 20 days with a computer with a processor Intel Core i7-870 $2.93 \mathrm{GHz}$ with four cores. Such a (simulated) $Q-V$ sample was divided into the model selection sample with $n_{\text {sel }}=2000(Q, V)$ pairs and the simulated validation sample with $n_{\mathrm{val}}=7000(Q, V)$ pairs. Their scatter plots are shown in Fig. 4. The Kendall's $\tau$ of both samples is 0.7 with a suitable $p$-value less than 0.05 , indicating a positive dependence relation between variables.

\subsubsection{Sensitivity analysis: minimum data length needed}

The sensitivity analyses to identify the minimum sample length required for obtaining robust quantile estimates by each marginal distribution, in terms of the uncertainty of the quantile estimate by using the whole univariate either $Q$ or $V$ series, are shown in Fig. 5. The 100-year quantile was selected in this study (named as $\hat{q}_{100}$ ) as a trade-off between a high enough quantile and a quantile not entailing a large uncertainty. Following the procedure explained in Sect. 2.2, the box plots of the quantiles obtained from the bootstrap samples (from the model selection sample) of each length $n$ are plotted along the $x$-axis. The boundaries associated with the confidence interval for the model selection sample are added as two horizontal lines. The minimum data length, $n_{\mathrm{m}}$, for which the confidence interval (i.e., the borders of the box) is inside such boundaries, i.e., $n_{\mathrm{m}, Q}$ and $n_{\mathrm{m}, V}$, are marked by a dotted line in Fig. 5 for each marginal distribution. As expected, the $n_{\mathrm{m}}$ required in the case of the two-parameter $\mathrm{G}$ distribution is less than that needed for the three-parameter (GEV, GLO and LNO) distributions. Moreover, the quantile related to $Q$ requires the same or a similar data length than that related to $V$ for each marginal distribution, with the exception of the GLO distribution for which the difference is larger (due to the randomness of the process and the data step considered).

The results of the sensitivity analyses of the copula parameter estimate are shown in Fig. 6. In this case, the objective of the analysis is to identify the minimum record length, $n_{\mathrm{c}}$, required for assuming that the parameter estimated for each copula is robust enough in reference to
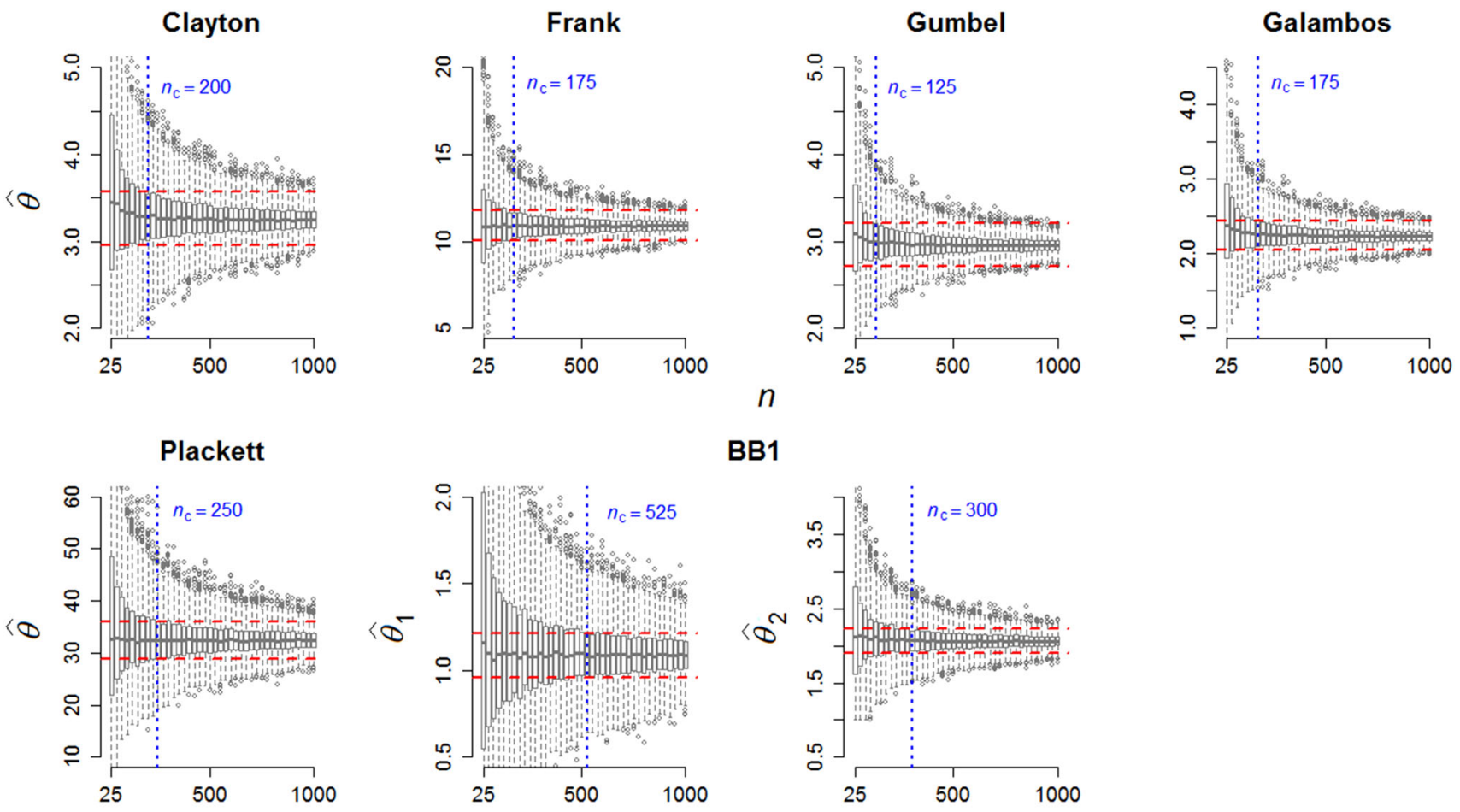

BB1

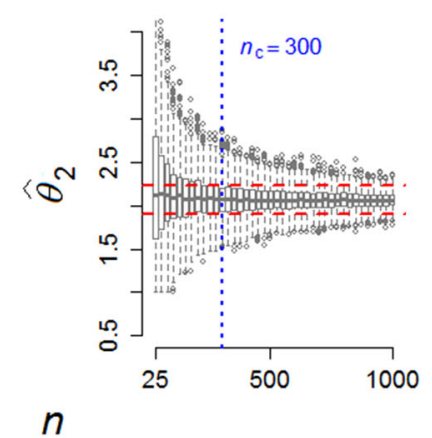

z: : boundaries related to model selection sample

Fig. 6 Sensitivity analysis for identifying the minimum sample length required for each copula. The box plot of the copula parameter(s) estimated from the bootstrap samples of length $n$ is plotted along the $x$-axis, where the points represent the outliers 


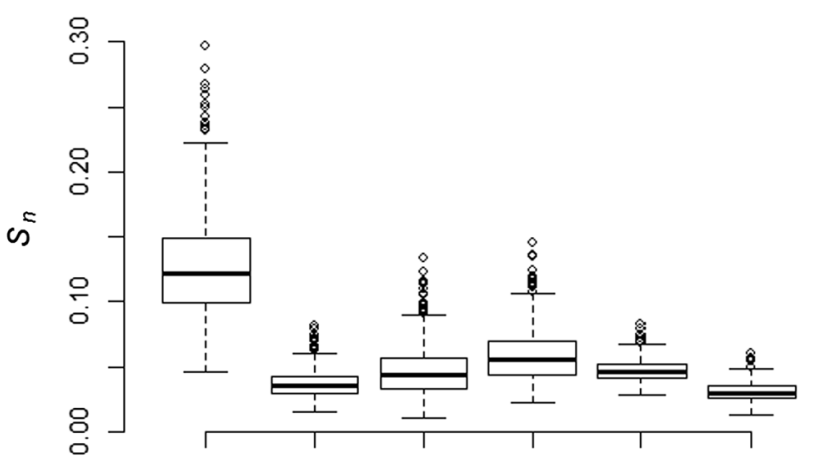

(a)

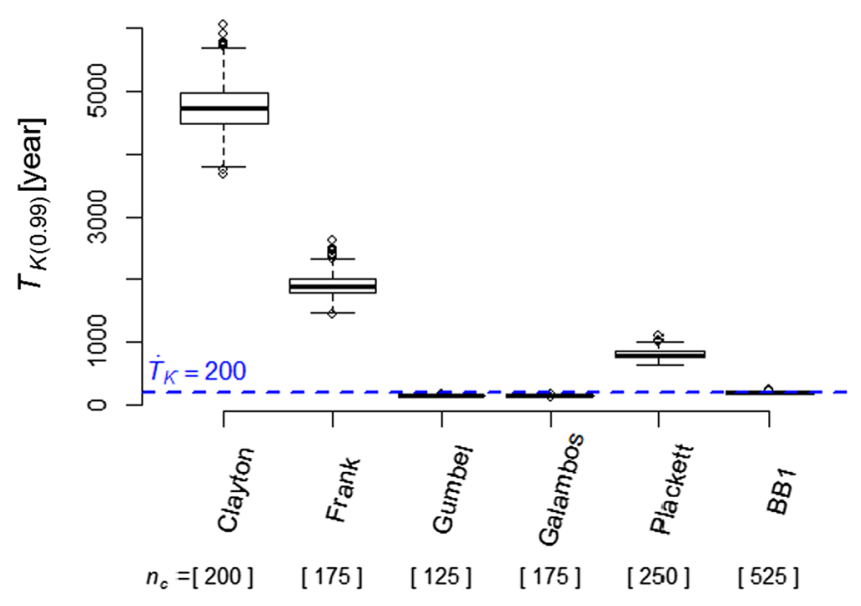

(c)

Fig. 7 Copula selection process based on 1000 bootstrap samples with a sample length $n_{\mathrm{c}}$ for each copula. Box plots for each copula show a the assessment of the fit to the data via $S_{n}$; $\mathbf{b}$ the results of the formal goodness-of-fit test by the $p$-value of $S_{n}$; $\mathbf{c}$ the adequacy of the

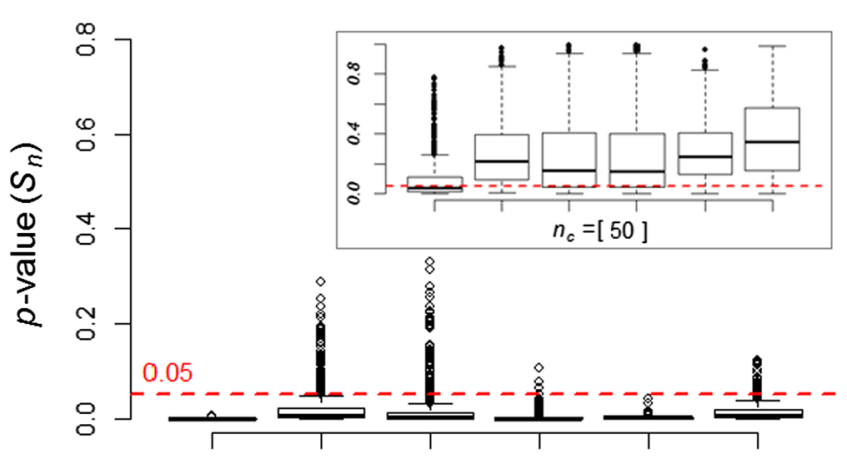

(b)

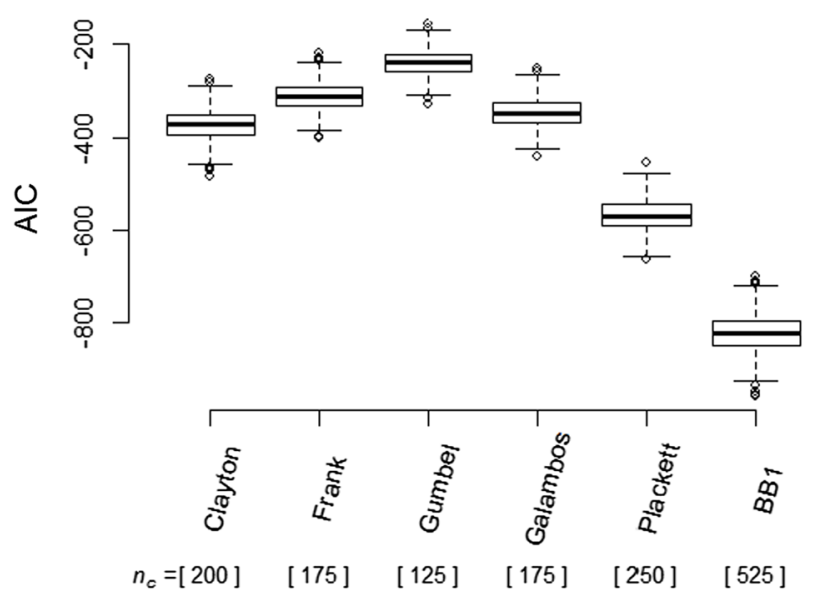

(d)

Kendall's return period $T_{K(0.99)}$ in reference to the empirical Kendall's return period $\dot{T}_{K(0.99)}$; and $\mathbf{d}$ the evaluation of the AIC for ranking copulas. The points in the box plots represent the outliers
Fig. 8 Comparison among the empirical estimate of the Kendall's function (for $t$ copula values) and the theoretical estimate, regarding the model selection sample, obtained by a the Clayton, Frank and Gumbel copula; and b the Galambos, Plackett and BB1 copula. Results are divided into two figures for clarity

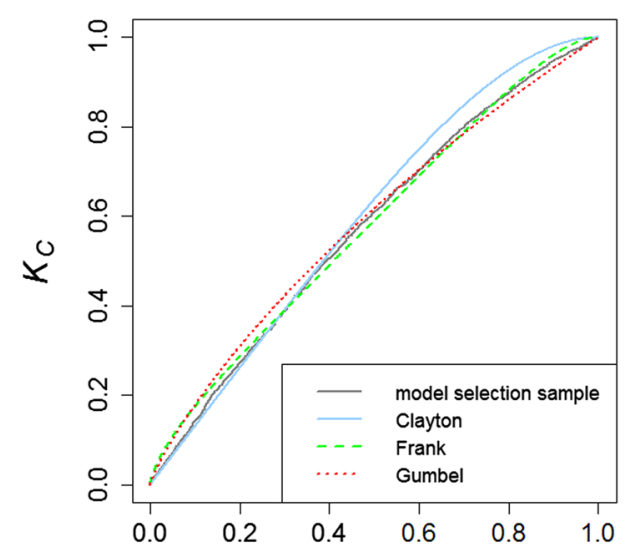

(a)

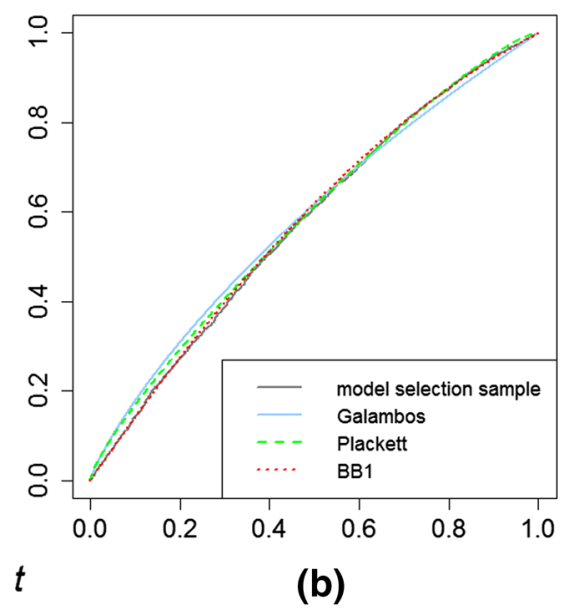

(b) uncertainty of the parameter estimate via the whole bivariate $Q-V$ series. Analogously to the univariate case, the required $n_{\mathrm{c}}$, identified as the minimum data length for which the confidence interval is less than the boundaries associated with the confidence interval of the copula parameter for the model selection sample (i.e., the two horizontal lines), is marked in Fig. 6 for each copula. Note that for the BB1 copula $n_{\mathrm{c}}=525$, which is the maximum of the two values obtained by applying the procedure to each copula parameter. As expected, the $n_{\mathrm{c}}$ needed for the 

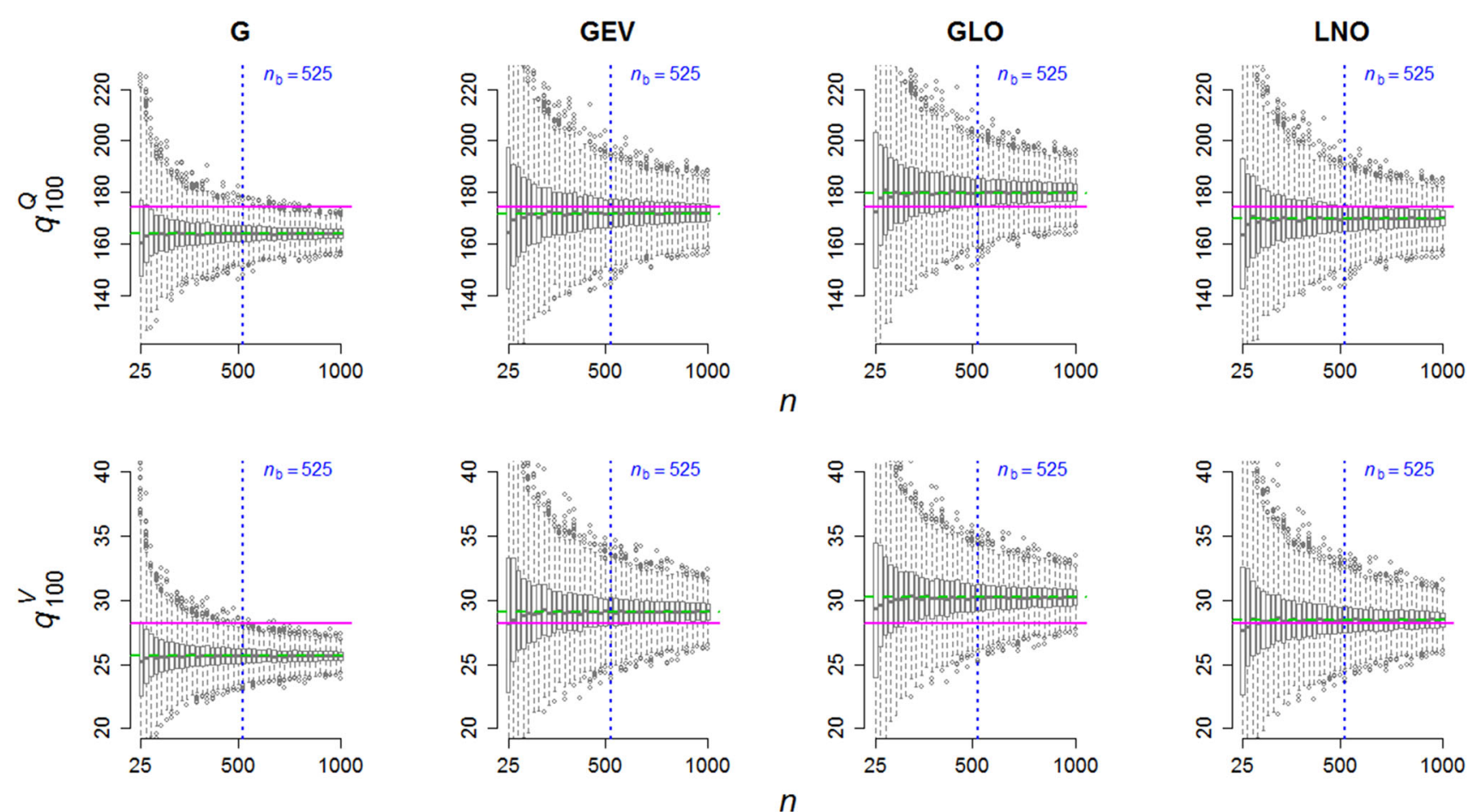

$-\cdots \hat{q}_{T}-\dot{q}_{T}$

Fig. 9 Selection of the marginal distributions (for $Q$ and $V$ ) based on 1000 bootstrap samples from the model selection sample. Box plots show the variability of the univariate quantile estimated for $T=100$ years, $\hat{q}_{100}$, plotting its median value to be compared with

two-parameter copula BB1 is greater than that needed for the rest of the one-parameter (Clayton, Frank, Gumbel, Galambos and Plackett) copulas. Although in general and as expected, the minimum record length for a copula is greater than that needed for a marginal distribution, the value associated with the Gumbel copula is less than those associated with some marginal distributions.

\subsubsection{Identification of the bivariate model based on copulas}

The identification of the bivariate distribution of $Q$ and $V$, requiring the selection of the marginal distributions and the copula, is conducted by the procedure detailed in Sect. 2.3. First, the results obtained for the copula selection process are shown in Fig. 7. The assessment of the fit of each copula to the data is displayed in Fig. 7a, via the box plot of the $S_{n}$ values obtained by Eq. 2, considering the corresponding bootstrap sample of length $n_{\mathrm{c}}$ obtained previously. As a result, the Clayton copula was identified as the worst copula in terms of fitting to the data, as both the median value of the $S_{n}$ and its variability represented by the height of the box are the largest. The values obtained for the rest of the copulas are smaller and similar to each other, obtaining the best results its median empirical quantile estimate, $\dot{q}_{100}$, for bootstrap samples with a sample length equal to $n_{\mathrm{m}}$. Points in the box plots represent the outliers

Table 1 Relative error $(R E)$ of the (median) quantile estimated for 1000 bootstrap samples of length $n_{\mathrm{b}}=525$

\begin{tabular}{llr}
\hline Marginal distribution & \multicolumn{2}{l}{$R E(\%)$ for $n_{\mathrm{b}}=525$} \\
\cline { 2 - 3 } & \multicolumn{2}{l}{$V$} \\
\hline G & -5.9 & -9.0 \\
GEV & $\mathbf{- 1 . 4}$ & 3.2 \\
GLO & 3.2 & 7.1 \\
LNO & -2.6 & $\mathbf{1 . 0}$ \\
\hline
\end{tabular}

for the Frank and BB1 copula. Consequently, the Clayton copula is discarded, as it is not able to represent the dependence between the observed $Q-V$ pairs.

The box plots of the $p$-values associated with the $S_{n}$ values are displayed in Fig. 7b. As expected, because of the use of large sample lengths (see Sect. 2.3.1 for more details), poor results were obtained by the goodness-of-fittest. Only some $p$-values greater than 0.05 , where such a threshold is indicated as a horizontal line, were obtained as outliers for the Frank, Gumbel, Galambos and BB1 copula. The results for a smaller sample length $\left(n_{\mathrm{c}}=50\right)$, which is in the range of the common observed data lengths recorded 
Fig. 10 a Fit of the marginal distributions of $Q$ and $V$ to the model selection sample;

b Comparison between the flood frequency curve fitted to the simulated validation sample generated through the hydrometeorological model, and the confidence interval obtained by the synthetic validation samples generated by the bivariate distribution
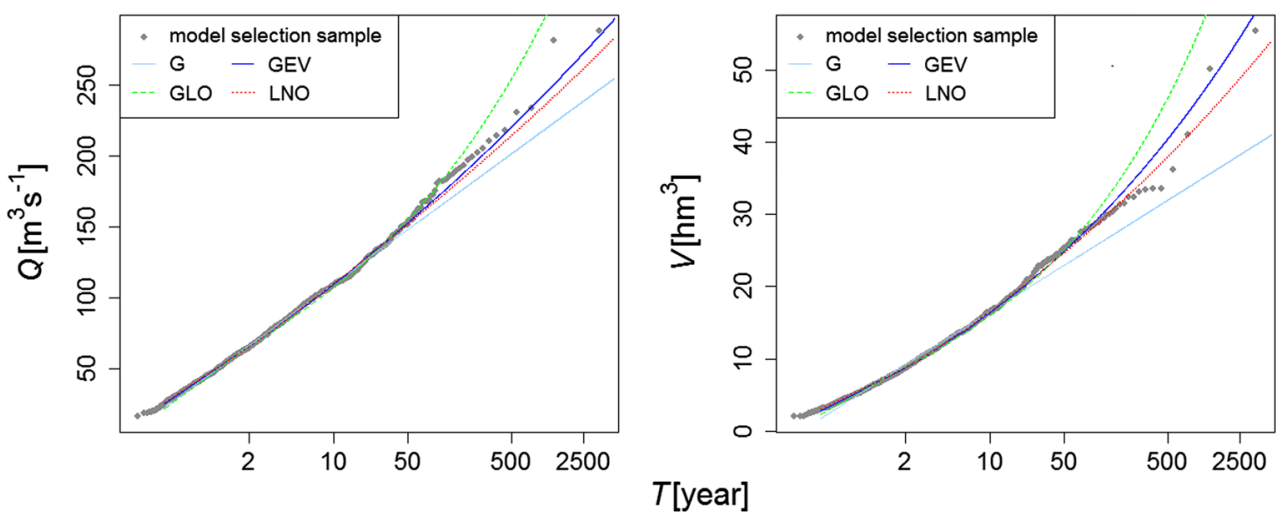

GEV

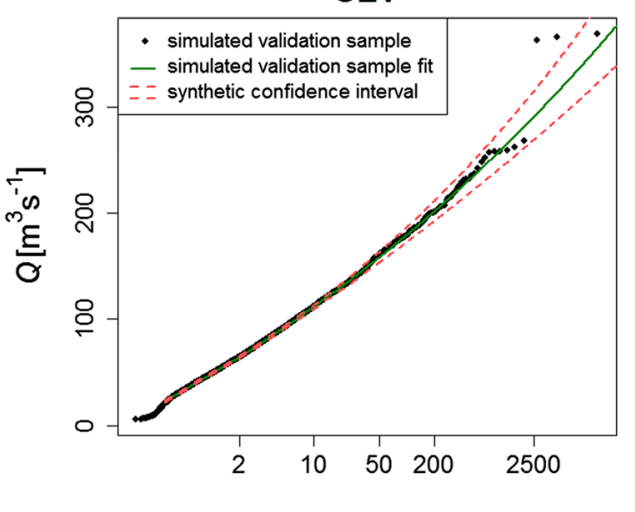

(a)

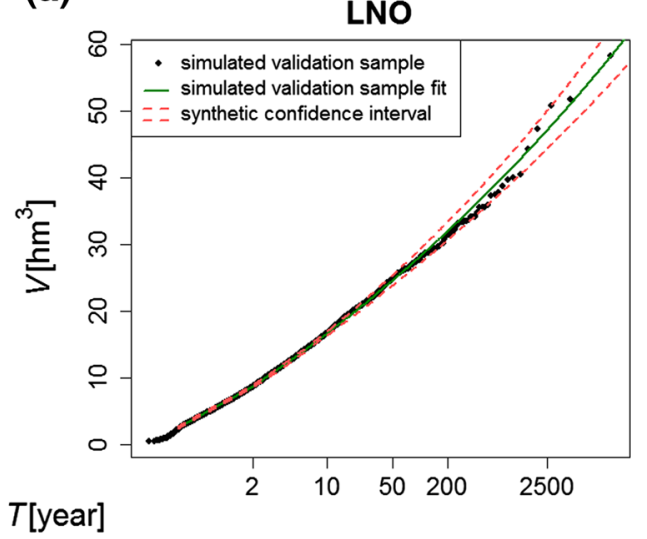

(b)
Table 2 Confidence interval (in percentage) of the flood frequency curves obtained via 10,000 synthetic validation samples, for $T=10,25,100$ and 500 years

\begin{tabular}{|c|c|c|c|c|c|}
\hline \multirow[t]{2}{*}{ Variable } & \multirow[t]{2}{*}{ Marginal distribution } & \multicolumn{4}{|c|}{ Confidence interval range (\%) for $T$} \\
\hline & & 10 & 25 & 100 & 500 \\
\hline$Q$ & GEV & \pm 1.8 & \pm 2.5 & $(-3.8,+3.9)$ & $(-5.5,+6.0)$ \\
\hline$V$ & LNO & \pm 2 & \pm 2.7 & \pm 3.7 & $(-4.9,+5.0)$ \\
\hline
\end{tabular}

in practice (see Sect. 1), are also plotted in the upper right corner of Fig. 7b for illustration purposes. As expected, more suitable $p$-values were obtained. All copulas except the Clayton copula pass the goodness-of-fit test in this case. Consequently, the $p$-value is not used for identifying the suitable copulas in this study.

The adequacy of the Kendall's return period estimate for a high copula value $t=0.99, T_{K(0.99)}$ (Eq. 4), is analysed in Fig. 7c. The box plot of $T_{K(0.99)}$ for each copula is displayed together with the empirical value associated with the whole model selection sample as it was indicated in Sect. 2.3.1, $\dot{T}_{\mathrm{K}(0.99)}=200$ years, which is plotted as a horizontal line. It can be seen that besides the already discarded Clayton copula in terms of $S_{n}$ results, the Frank and Plackett copula show a large overestimate of the empirical value (in decreasing order). The Clayton and Frank copula also show a larger variability. Closer estimates to the empirical value, involving underestimate, were obtained by the Gumbel, Galambos and BB1 copula. For the first two copulas $T_{K(0.99)}=150$ years, while for the BB1 copula $T_{K(0.99)}=192$ years, being the last the best estimate. Note that an underestimate of the return period entails being on the safety side. As a result, the Frank and Plackett copulas are also discarded.

The results obtained by the AIC (Eq. 5) are shown in Fig. 7d. As it can be seen, the sample length affects the results, obtaining better values those copulas considering larger lengths. As a consequence, AIC results were obtained by using the same sample length for all copulas (not shown), resulting the BB1 copula the best copula in all cases. Hence, considering all the information provided by the copula selection process, the BB1 copula was chosen as the best copula for characterising the $Q-V$ series, requiring a minimum sample length $n_{\mathrm{c}}^{*}=525$. A visual support of the behaviour of the copulas regarding the model selection sample (with $n_{\text {sel }}=2000$ ), by the comparison of the 
theoretical Kendall's function of each copula and the empirical estimate, is plotted as an example in Fig. 8. It can be seen that the BB1 copula (Fig. 8b) involves the best fit.

As $n_{c}^{*}$ is greater than any $n_{\mathrm{m}}$ (see Fig. 5), $n_{\mathrm{b}}=n_{\mathrm{c}}^{*}=525$ and hence, the selection of the marginal distributions is conducted under 1000 bootstrap samples of such a length, estimating the (median) quantile $\hat{q}_{100}$ for each marginal distribution, as well as the (median) empirical quantile $\dot{q}_{100}$. The distances between both quantiles are plotted in Fig. 9, and the $R E$ values obtained via Eq. 6 are shown in Table 1. It was found that the best marginal distributions are the GEV for $Q$ and the LNO for $V$, as they entail the minimum absolute value of $R E$ in each case. The fit of the marginal distributions to the model selection sample is plotted in Fig. 10a as an illustration of the behaviour of the marginal distributions. It can be seen that the fit of the GEV distribution is the closest to the peak flow data, $Q$, and the fit of the LNO distribution is the closest to the volume data, $V$. In summary, the bivariate distribution selected to represent the $Q-V$ series consists of the BB1 copula, the GEV distribution for $Q$ and the LNO distribution for $V$, with a sample length of $n_{\mathrm{b}}=525$.

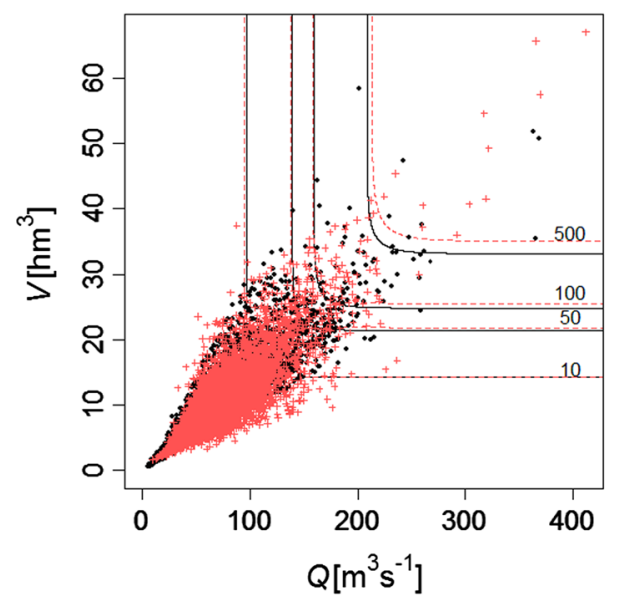

$\begin{array}{lll}- & \text { simulated } T_{K} \text { curves } \\ \text { synthetic } T_{K} \text { curves }\end{array} \quad+\quad \begin{aligned} & \text { simulated validation sample } \\ & \text { synthetic validation sample }\end{aligned}$

Fig. 11 Comparison between the Kendall's return period curves estimated by the simulated validation sample generated through the hydro-meteorological model, and by a given synthetic sample generated by the bivariate distribution

\subsubsection{Validation of the methodology}

The methodology is then validated according to Sect. 2.4. Synthetic validation samples (of size $n_{\text {val }}=7000$ ) are generated by fitting the selected bivariate distribution to bootstrap samples of the identified length $\left(n_{\mathrm{b}}=525\right)$, obtained without replacement from the simulated validation sample. Regarding the marginal distributions, the flood frequency curve of the simulated validation sample is drawn together with the confidence interval related to the flood frequency curves of 10,000 synthetic validation samples (Fig. 10b). The flood frequency curve is tightly fitted by the confidence interval for small $T$ values. The range slightly increases for larger values of $T$, as the uncertainty is larger. The simulated data remain inside the synthetic confidence interval in the case of $V$, and only two data points are outside in the case of $Q$, as the three largest peaks show similar values. The confidence interval in percentage is shown in Table 2 for several $T$ values. The confidence intervals associated with the copula level curves of 1000 synthetic validation samples are almost equal to the corresponding curves obtained via the simulated validation sample (not shown).

As an example of the results obtained by the application of the methodology, the simulated validation sample is plotted together with a given synthetic validation sample in Fig. 11. The Kendall's return period curves estimated by the bivariate distribution fitted to each sample are also displayed (estimated parameters shown in Table 3). It can be seen that both scatter plots are visually similar and that the results regarding the Kendall's return period curves are comparable. The Kendall's return period curves are practically identical for small Kendall's return period values, while as expected, such a difference becomes slightly larger the larger the return period value is, because of the increasing uncertainty.

\section{Conclusions}

In the present paper a bivariate procedure to extend flood series due to the need of achieving more appropriate flood frequency analyses is addressed, determining the minimum number of flood hydrographs required to be simulated by a hydro-

Table 3 Parameters of the fitted bivariate distribution (marginal distributions and copula) for the simulated validation sample, and a given bootstrap sample of size $n_{\mathrm{b}}=525$ that belongs to the simulated validation sample, whereby the synthetic validation sample is generated

\begin{tabular}{|c|c|c|c|c|c|c|c|c|}
\hline \multirow[t]{2}{*}{ Validation sample } & \multicolumn{3}{|c|}{ GEV distribution for $Q$} & \multicolumn{3}{|c|}{ LNO distribution for $V$} & \multicolumn{2}{|c|}{ BB1 copula } \\
\hline & Location & Scale & Shape & Location & Scale & Shape & $\hat{\theta}_{1}$ & $\hat{\theta}_{2}$ \\
\hline Simulated & 55.939 & 23.011 & -0.065 & 8.788 & 4.441 & -0.497 & 1.132 & 2.035 \\
\hline Synthetic & 55.662 & 21.771 & -0.095 & 8.836 & 4.401 & -0.551 & 1.178 & 1.933 \\
\hline
\end{tabular}


meteorological model to be used as input for obtaining an extended flood series by a bivariate model based on copulas.

A previously calibrated distributed hydro-meteorological model is used for simulating a series of flood hydrographs, with the aim of extending the observed peak-volume series by a bivariate distribution consisting of two marginal distributions and a copula. The minimum data length needed to be simulated by the hydro-meteorological model is defined through a sensitivity analysis in order to obtain robust estimates from both marginal distributions and copula. The marginal distribution and copula selection process is performed, where the copula selection process is carried out by taking into account the fit of the copula to the data, the adequacy of high joint return period estimates (using the Kendall's return period), and the results of a model selection criterion. As a result, the selected bivariate distribution fitted to a small sample simulated by the hydro-meteorological model is used for generating arbitrarily large synthetic samples. The adequacy of the procedure is checked by comparing the flood frequency curve (of each marginal distribution) and the copula level curves fitted to a large sample simulated by the hydro-meteorological model, with the corresponding confidence intervals obtained from a large amount of synthetic samples generated by the bivariate distribution.

The proposed methodology was applied to the Santillana reservoir gauging station in the Manzanares River located in Spain. It was found that a minimum data length of 525 flood hydrographs should be simulated through the hydrometeorological model in order to accomplish a robust fit by a bivariate distribution based on the two-parameter BB1 copula, which was chosen as the best copula by the copula selection process. In this regard, it is suggested considering the BB1 copula as potential candidate for characterising peak-volume series in other catchments. As expected, a smaller data length (in the order of 200 data) should be required in the case of a one-parameter copula was selected. The generalised extreme value distribution for the peak flow and the log-normal distribution for the hydrograph volume were found to be the best marginal distributions for a record length of 525 years. As a result, large synthetic samples were stochastically generated by fitting the bivariate distribution to a random set of 525-length samples simulated by the hydro-meteorological model. The comparable performance of such synthetic samples in relation to a sample of the same length simulated by the hydrometeorological model supported the use of the proposed methodology. The procedure provides an extended sample composed of 525 data from the simulation through a hydrometeorological model and a much larger synthetic sample stochastically generated by fitting the bivariate distribution.

The proposed procedure allows cutting down the computation time required for generating a large sample of peak-volume pairs, in comparison to the time needed by a hydro-meteorological modelling chain (specifically, from a month scale to few days for the sample length generated for the case study), allowing the generation of a peak-volume sample as long as desired to enable more suitable flood risk assessment studies.

Acknowledgments This work has been supported by the Carlos González Cruz Foundation and the project 'MODEX-Physicallybased modelling of extreme hydrologic response under a probabilistic approach. Application to Dam Safety Analysis' (CGL2011-22868), funded by the Spanish Ministry of Science and Innovation (now the Ministry of Economy and Competitiveness). The Authors would like to thank two anonymous reviewers for the useful comments provided to improve the quality of the paper.

\section{References}

Beven KJ (1987) Towards the use of catchment geomorphology in flood frequency predictions. Earth Surf Proc Land 12:69-82

Blazkova S, Beven KJ (1997) Flood frequency prediction for data limited catchments in the Czech Republic using stochastic rainfall model and TOPMODEL. J Hydrol 195:256-278

Blazkova S, Beven K (2004) Flood frequency estimation by continuous simulation of subcatchment rainfalls and discharges with the aim of improving dam safety assessment in a large basin in the Czech Republic. J Hydrol 292:153-172

Bonta JV, Rao AR (1988) Factors affecting the identification of independent storm events. J Hydrol 98:275-293

Boughton W, Droop O (2003) Continuous simulation for design flood estimation-a review. Environ Model Softw 18:309-318

Boughton W, Srikanthan S, Weinmann E (2002) Benchmarking a new design flood estimation system. Aust J Water Resour 6(1):45-52

Burton A, Kilsby C, Fowler H, Cowpertwait P, O'Connell P (2008) RainSim: a spatial-temporal stochastic rainfall modelling system. Environ Model Softw 23:1356-1369

Cabral MC, Garrote L, Bras RL, Entekhabi D (1992) A kinematic model of infiltration and runoff generation in layered and sloped soils. Adv Water Resour 15:311-324

Calver A, Lamb R (1995) Flood frequency estimation using continuous rainfall-runoff modelling. Phys Chem Earth 20:479-483

Cameron DS, Beven KJ, Tawn J, Blazkova S, Naden P (1999) Flood frequency estimation by continuous simulation for a gauged upland catchment (with uncertainty). J Hydrol 219:169-187

Candela A, Brigandì G, Aronica G (2014) Estimation of synthetic flood design hydrographs using a distributed rainfall-runoff model coupled with a copula-based single storm rainfall generator. Nat Hazard Earth Syst 14:1819-1833

Chebana F, Ouarda TBMJ (2011) Multivariate quantiles in hydrological frequency analysis. Environmetrics 22:63-78

Chetty K, Smithers J (2005) Continuous simulation modelling for design flood estimation in South Africa: preliminary investigations in the Thukela catchment. Phys Chem Earth 30:634-638

Chowdhary H, Escobar LA, Singh VP (2011) Identification of suitable copulas for bivariate frequency analysis of flood peak and flood volume data. Hydrol Res 42:193-216

Cowpertwait PS (1994) A generalized point process model for rainfall. Proc R Soc Lond A 447(1929):23-37

Cowpertwait PS (1995) A generalized spatial-temporal model of rainfall based on a clustered point process. Proc R Soc Lond A 450(1938):163-175

Cunnane C (1989) Statistical distributions for flood frequency analysis. World Meteorological Organization, Geneva, Switzerland, Operational Hydrology Report, pp 23-33 
De Michele C, Salvadori G, Canossi M, Petaccia A, Rosso R (2005) Bivariate statistical approach to check adequacy of dam spillway. J Hydrol Eng 10:50-57

Eagleson P (1972) Dynamics of flood frequency. Water Resour Res 8(4):878-898

Engeland K, Xu C, Gottschalk L (2005) Assessing uncertainties in a conceptual water balance model using Bayesian methodology. Hydrol Sci J 50:45-63

England JF Jr, Velleux ML, Julien PY (2007) Two-dimensional simulations of extreme floods on a large watershed. J Hydrol 347:229-241

Favre AC, El Adlouni S, Perreault L, Thiémonge N, Bobée B (2004) Multivariate hydrological frequency analysis using copulas. Water Resour Res 40:W01101

Flores I, Sordo-Ward A, Mediero L, Garrote L (2013) Deriving bivariate flood frequency distributions for dam safety evaluation. In Proceedings of EWRA 2013: water resources management in an interdisciplinary changing context: Session 4, 93

Garrote L, Bras RL (1995a) A distributed model for real-time flood forecasting using digital elevation models. J Hydrol 167: 279-306

Garrote L, Bras RL (1995b) An integrated software environment for real-time use of a distributed hydrologic model. J Hydrol 167: 307-326

Genest C, Favre AC (2007) Everything you always wanted to know about copula modeling but were afraid to ask. J Hydrol 12: 347-368

Genest C, Remillard B (2008) Validity of the parametric bootstrap for goodness-of-fit testing in semiparametric models. Annales De L'Institut Henri Poincare-Probabilites Et Statistiques 44:1096-1127

Genest C, Rivest L (1993) Statistical inference procedures for bivariate archimedean copulas. J Am Stat Assoc 88:1034-1043

Genest C, Ghoudi K, Rivest L (1995) A semiparametric estimation procedure of dependence parameters in multivariate families of distributions. Biometrika 82:543-552

Genest C, Quessy JF, Rémillard B (2006) Goodness-of-fit procedures for copula models based on the probability integral transformation. Scand J Stat 33:337-366

Genest C, Rémillard B, Beaudoin D (2009) Goodness-of-fit tests for copulas: a review and a power study. Insur Math Econ 44:199-213

Giustarini L, Camici S, Tarpanelli A, Brocca L, Melone F, Moramacro T (2010) Dam spillways adequacy evaluation through bivariate flood frequency analysis and hydrological continuous simulation. Conference proceedings of the Word Environmental and Water Resources Congress 2010: Challenges of Change, ASCE

Goel N, Seth S, Chandra S (1998) Multivariate modeling of flood flows. J Hydraul Eng-ASCE 124:146-155

GREHYS (1996) Presentation and review of some methods for regional flood frequency analysis. J Hydrol 186:63-84

Joe H (1997) Multivariate model and dependence concepts. Chapman and Hall, London

Klein B, Pahlow M, Hundecha Y, Schumann A (2010) Probability analysis of hydrological loads for the design of flood control systems using copulas. J Hydrol Eng 15:360-369

Kuchment LS, Gelfan AN, Demidov VN (2003) Application of dynamic-stochastic runoff generation models for estimating extreme flood frequency distributions. IAHS-AISH P 281: $107-114$

Lawrence D, Paquet E, Gailhard J, Fleig AK (2014) Stochastic semicontinuous simulation for extreme flood estimation in catchments with combined rainfall-snowmelt flood regimes. Nat Hazard Earth Sys 14:1283-1298
Mediero L, Garrote L, Martín-Carrasco F (2011) Probabilistic calibration of a distributed hydrological model for flood forecasting. Hydrol Sci J 56:1129-1149

Paquet E, Garavaglia F, Garçon R, Gailhard J (2013) The SCHADEX method: a semi-continuous rainfall-runoff simulation for extreme flood estimation. J Hydrol 495:23-37

Requena AI, Mediero L, Garrote L (2013) A bivariate return period based on copulas for hydrologic dam design: accounting for reservoir routing in risk estimation. Hydrol Earth Syst Sc 17:3023-3038

Requena AI, Chebana F, Mediero L (2015a) A complete procedure for multivariate index-flood model application. J Hydrol. (under review)

Requena AI, Prosdocimi I, Kjeldsen TR, Mediero L (2015b) A bivariate trend analysis to investigate the effect of increasing urbanisation on flood characteristics. Hydrol Res. (under review)

Restrepo-Posada PJ, Eagleson PS (1982) Identification of independent rainstorms. J Hydrol 55:303-319

Saad C, El Adlouni S, St-Hilaire A, Gachon P (2015) A nested multivariate copula approach to hydrometeorological simulations of spring floods: the case of the Richelieu River (Québec, Canada) record flood. Stoch Environ Res Risk Assess 29:275-294

Salvadori G, De Michele C (2004) Frequency analysis via copulas: theoretical aspects and applications to hydrological events. Water Resour Res 40:W12511

Salvadori G, De Michele C (2010) Multivariate multiparameter extreme value models and return periods: a copula approach. Water Resour Res 46:W10501

Salvadori G, De Michele C, Kottegoda NT, Rosso R (2007) Extremes in nature: an approach using copulas. Springer, Dordrecht

Salvadori G, De Michele C, Durante F (2011) On the return period and design in a multivariate framework. Hydrol Earth Syst Sc 15:3293-3305

Serinaldi F, Grimaldi S (2007) Fully nested 3-copula: procedure and application on hydrological data. J Hydrol Eng 12:420-430

Shiau J, Wang H, Tsai C (2006) Bivariate frequency analysis of floods using copulas. J Am Water Resour As 42:1549-1564

Sklar A (1959) Fonctions de répartition à $\mathrm{n}$ dimensions et leurs marges. Publ Inst Statist Univ Paris 8:229-231

Song S, Singh VP (2010) Frequency analysis of droughts using the Plackett copula and parameter estimation by genetic algorithm. Stoch Environ Res Risk Assess 24(5):783-805

Vandenberghe S, Verhoest NEC, De Baets B (2010) Fitting bivariate copulas to the dependence structure between storm characteristics: a detailed analysis based on 105 year 10 min rainfall. Water Resour Res 46:W01512

Vrugt JA, Bouten W, Gupta HV, Sorooshian S (2002) Toward improved identifiability of hydrologic model parameters: the information content on experimental data. Water Resour Res 38(12):48-1-48-13

Yue S, Ouarda T, Bobée B, Legendre P, Bruneau P (1999) The Gumbel mixed model for flood frequency analysis. J Hydrol 226:88-100

Zhang L, Singh VP (2006) Bivariate flood frequency analysis using the copula method. J Hydrol Eng 11:150-164

Zhang L, Singh VP (2007) Trivariate flood frequency analysis using the Gumbel-Hougaard copula. J Hydrol Eng 12:431-439

Zhang Q, Xiao M, Singh VP, Chen X (2013) Copula-based risk evaluation of hydrological droughts in the East River basin. China. Stoch Environ Res Risk Assess 27(6):1397-1406 